\title{
ASYMPTOTIC LOCATION OF NODAL LINES USING GEODESIC THEORY
}

\author{
J. J MAHONY ${ }^{1}$ AND J. NORBURY ${ }^{2}$
}

(Received 17 July 1984; revised 20 May 1985)

\begin{abstract}
The asymptotic propertues of solutions of the non-linear eigenvalue problem, associated with the homogeneous Dirichlet problem for

$$
\varepsilon^{2} \nabla^{2} u=-f^{2}\left(g^{2}-u^{2}\right) u,
$$

are investigated. Here $f$ and $g$ are smooth functions of position in a finite plane region with a smooth boundary. The results for the positive solution are well established, but knowledge of other branches of solutions is scarce. Here positive solutions are pieced together across lines partitioning the domain, and variational arguments are framed, as an effective means of locating the lines, so that the composite function is everywhere a solution of *. Heuristic arguments suggest strongly that there is a close relationship between the nodal lines of * and certain classes of weighted geodesic lines defined by the classical variational problem for the functional

$$
\oint f g^{3} d s
$$

which provides an effective basis for computation. Some results are proved but others remain conjectures. Analogous results are proved for the associated ordinary differential equation. The geometry of the solutions is surprisingly restricted when the coefficients are spatially variable. The arguments are extended to a class of reactive, diffusive systems. It is possible to predict the pattern of domains of different outcomes in terms of properties of the surface on which the reactions occur, without a knowledge of the chemical kinetics. The results appear to provide a basis for stringent testing of the postulated role of reactive-diffusive mechanisms in the formation of complex patterns in biological species.
\end{abstract}

\section{Introduction}

Determination of the asymptotic properties of branches of solutions of non-linear eigenvalue problems has proved to be very much more difficult than might have

\footnotetext{
${ }^{1}$ Department of Mathematics, University of Western Australia, Nedlands, W. A. 6009.

2St. Catherine's College, Oxford.

(c) Copyright Australian Mathematical Society 1986, Serial-fee code 0334-2700/86
} 
been expected initially. As an illustration, consider the following Dirichlet problem

$$
\varepsilon^{2} \nabla^{2} u=-f^{2}\left(g^{2}-u^{2}\right) u, \quad \mathbf{x} \in D,
$$

subject to the homogeneous Dirichlet boundary condition

$$
u=0, \quad \mathrm{x} \in \partial D \text {. }
$$

The domain $D$ is a simply connected plane region with a smooth boundary $\partial D$. Here both $f$ and $g$ are given smooth functions of $\mathbf{x}$, both are strictly positive and $\varepsilon$ is a positive parameter. Apart from the trivial solution $u=0$, there exists a number of more interesting solutions to this Dirichlet problem. Each eigenvalue of the Dirichlet problem for the associated linear equation

$$
\nabla^{2} w=-\lambda f^{2} g^{2} w
$$

defines a bifurcation point from which arise branches of solutions of the nonlinear problem.

The parameter $\varepsilon$ serves to locate position on any such brancn, and smallness of the parameter may be used to describe the state far from the bifurcation point. For only one branch, namely that originating at the lowest eigenvalue $\lambda_{0}$, is any significant knowledge of the asymptotics available. For any given value of $\varepsilon$, the solution on that particular branch is both

(1) the only positive solution, and

(2) the unique global minimiser, within the function class $\dot{W}_{1,2}(D)$, of the energy functional,

$$
E(v, D)=\int\left\{\varepsilon^{2}(\nabla v)^{2}+\frac{1}{2} f^{2}\left(g^{2}-v^{2}\right)^{2}\right\} .
$$

Hereafter the function class $\dot{W}_{1,2}(D)$ will be denoted by $H$. Thus there is a number of very powerful techniques available for the investigation of the properties of solutions on this branch. Berger and Fraenkel [1] established the asymptotic properties of this positive solution for a slightly less general equation where the product $f g$ is unity. They were forced to make strong smoothness assumptions for both the coefficients and the boundary. While their assumptions seem inappropriately strong, we make corresponding assumptions here in order to be able to use their results for the asymptotic properties of positive solutions. We transfer their results to the more general equation $(1,1 \mathrm{a})$. A detailing of the small variations of the extensive calculations involved does not appear warranted.

For the other branches of solutions, there are the results typically obtained by following the solution from bifurcation. But as secondary bifurcation cannot be ruled out, and as there also may be bifurcation from infinity, the continuation of such branches to any asymptotic limit which may exist is shrouded in mystery. About the only information available is some numerical work of Budden and Norbury [2] for rectangles. Heuristic analysis has failed to provide suggestions as 
to the behavior as a basis for more serious discussion of the properties of the solutions. Even more remarkable is the fact that a similar position holds for the simpler case of the corresponding ordinary differential equation.

This paper represents an attempt to obtain as much information as possible by a combination of approximation methods and rigorous proof for both ordinary and partial differential equations. Attention will be confined to the case of small $\varepsilon$ and, where further, $u$ is close to $g$ over almost all the domain. Not to add the second part of this statement would bring under consideration branches which had just bifurcated from the trivial solution. Such branches consist of small-amplitude oscillations for which current results apply. There is a very natural alternative description of solutions on other branches in terms of their nodal lines -i.e. the lines on which $u=0$. When the coefficients are analytic, the possibility that $u$ vanishes in a sub-domain can be excluded, since the solution is also analytic. The assumption of analyticity can be relaxed using the theory of elliptic equations, so that nodal lines are the only possible sets on which a classical solution may vanish. On any nodal line the normal derivative must be non-zero almost everywhere. For if it were not, then by the Cauchy-Kowalewski proof, using the nodal line as support, there would be a domain in which the solution is zero. Thus all solutions, of interest here, change sign on nodal lines which serve to partition the domain into regions in which $u$ is either strictly positive or strictly negative. The solution in each of these subdomains is thus $\pm P$, where $P$ is the unique positive solution for that subdomain.

Now any solution of the problem posed by equations $(1,1)$ is associated with a critical point of the energy functional $E(v, D)$. Any classical solution can also be generated from its nodal lines by placing appropriate arrangements of the positive solutions on each sub-domain multiplied by \pm 1 . Thus if the domain $D$ is partitioned by a suitable set of lines, a function $\phi$ can be obtained by a similar use of positive solutions on each subdomain. For each set of such lines, the function $\phi$ is an element of $H$, and the subset of $H$ corresponding to all such sets of lines contains all the classical solutions. A knowledge of the dependence of the energy functional on the domain would then in principle permit the variational problem to be transformed to one defined in terms of varying the nodal lines. In Section 2, this idea is detailed, and alternative directions of utilising the different variational principles are examined. In Section 3, asymptotic estimates of the "energy" of the positive solution on an arbitrary domain are used to estimate the functional for an assumed set of nodal lines. The arguments place strong limitations on the properties of lines which can be nodal lines. The question of attempting to complete the proofs is considered in Section 4. Success with the proofs is limited, but evidence accumulates that the heuristic results are largely correct and we are led to make a conjecture, the confirmation of which would cover a large percentage of cases. 
In Section 5 attention is turned to the similar problem posed for an equivalent ordinary differential equation, and it is shown that all the results, conjectured for the partial differential equation, hold in this case. It is established that zeros (the analogues of nodal lines) can lie only in very restricted zones and, as a consequence, the qualitative patterns of the solutions differ markedly from the patterns of the solution branches close to their bifurcation from the trivial solution. The results are crucially dependent on the spatial inhomogeneity imposed by the functions $f$ and $g$ and do not apply to the degenerate case where $f g^{3}$ is close to a constant function. Section 6 contains a discussion of the mathematical implications of the results obtained and conjectured and their possible extension to cases where there are a larger number of independent variables.

There is a close mathematical relation between the partial differential equation considered here and systems of equations relevant to the study of reactive-diffusive systems of interest in biology. It has been argued by Murray, [3], [4] among others, that surface patterns particular to biological species may be determined by the outcomes of reactive-diffusive systems rather than by detailed genetic coding. It is shown, in Section 7, for a class of kinetics of an unusual kind, that the ideas developed here might be useful in investigating the extent to which the mechanism is relevant. For the class of systems of partial differential equations, admitting a variational formulation, it is possible to predict the patterns which develop on a curved surface when different equilibrium states are possible. The method requires no detailed knowledge of the chemistry and is predictive in terms of data describing the surface geometry, which might be obtained. The comparison of theoretical predictions and experimental observations would appear to permit a much more extended examination as to whether reactive-diffusive mechanisms can suffice to fix complex patterns associated with biological species differentiation.

\section{Variational formulation}

Let $u$ and $w$ be any two elements of $H$ defined on an arbitrary domain $D$ with a smooth boundary $\partial D$. Let $v$ be the difference

$$
v=w-u \text {. }
$$

Then it is a routine calculation to show that

$$
\begin{aligned}
E(w)-E(u)= & 2 \varepsilon^{2} \int_{\partial D} v\left(\frac{\partial u}{\partial n}+\frac{\partial v}{\partial n}\right)-2 \int_{D} v\left\{\varepsilon^{2} \nabla^{2} u+f^{2}\left(g^{2}-u^{2}\right) u\right\} \\
& -2 \int_{D} v\left\{\varepsilon^{2} \nabla^{2} v+f^{2}\left(3 u^{2}-g^{2}\right) v\right\} \\
& -\int_{D} f^{2}\left(3 u v^{3} / 2+v^{4} / 2\right)
\end{aligned}
$$


From this result some needed results follow easily. Although they are standard they are collected here for convenience.

RESULT 1. A function $u$ is a solution of equations $(1,1 \mathrm{a} \& \mathrm{~b})$ if, and only if, the Fréchet derivative $E^{\prime}(u, D)=0$.

RESULT 2. Let $P(\mathbf{x}, D)$ denote the unique positive solution which is the global minimiser of $E(w, D)$. Then the Dirichlet problem for the equation

$$
\begin{gathered}
\varepsilon^{2} \nabla^{2} v+f^{2}\left(g^{2}-3 P^{2}\right) v=l, \quad \mathbf{x} \in D, \\
v=m(\mathbf{x}), \quad \mathbf{x} \in \partial D,
\end{gathered}
$$

permits a unique bounded solution, for arbitrary $m$ and $l$.

This result follows by observing that, if there were two solutions, equation (2,3a) admits of a non-trivial solution $w$ satisfying homogeneous boundary conditions. As $[E(P+w)-E(P)]$ is dominated then by

$$
(3 / 2) \int_{0} f^{2} P v^{3}
$$

it is possible to use $v=-w$ to contradict the properties of the positive solution. The Fredholm Alternative Theorem then implies the result with an important restriction. The bound is not specific and can depend adversely on $\varepsilon$ and parameters fixing the geometry of the boundary.

Let $L$ denote a smooth simple line which divides $D$ into two disjoint sub-domains. There are two cases, illustrated in Figures 1a, $1 \mathrm{~b}$.

(a)

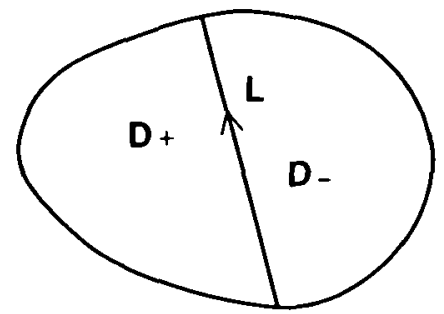

(b)

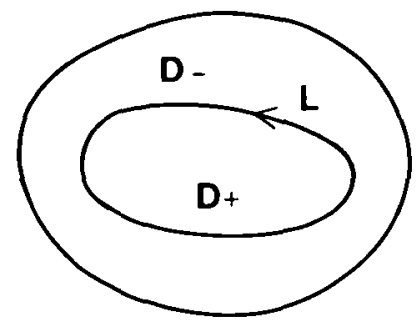

Figures la and $1 \mathrm{~b}$. The schematic partitioning of a domain $(D)$ into two sub-domains $\left(D_{+}\right.$and $D_{-}$) by a nodal line $L$.

Definition. Assign a sense of direction to the line $L$ (it does not matter which) and define a function $v$ by

$$
v(\mathrm{x})=\left\{\begin{array}{ll}
P\left(\mathrm{x}, D_{+}\right), & \mathrm{x} \in D_{+} \\
-P\left(\mathrm{x}, D_{-}\right), & \mathrm{x} \in D_{-}
\end{array}\right\},
$$

where the suffixes \pm respectively denote the sub-domains to the left and right of 
$L$. Then it is possible to associate with $L$ the functional $\mathscr{E}(L)$ defined by

$$
\mathscr{E}(L)=E(v, D)=E\left(P\left(\mathbf{x}, D_{+}\right)\right)+E\left(P\left(\mathbf{x}, D_{-}\right)\right) .
$$

Assign a unit normal $\mathbf{n}$ to $L$ directed from left to right.

The consideration of elements of the subset of $H$ constructed in this way, using a family of such lines, still serves to provide an adequate basis for testing for the vanishing of the Fréchet derivative $E^{\prime}(v)$. For, if another line $L^{\prime}$ is also considered, defining a function $w$, then the properties of the difference

$$
z=v-w
$$

can be obtained from the properties of the positive solutions. Thus

$$
\varepsilon^{2} \nabla^{2} z-f^{2}\left\{\left(g^{2}-3 P^{2}\right) z-3 v z^{2}-z^{3}\right\},
$$

where $P$ is the appropriate positive solution for the sub-domain. If the difference of the two functionals is calculated, using equation $(1,1)$ and the energy integral of equation $(2,5)$, the result

$$
\begin{aligned}
E(w)-E(v)= & \int\left\{2 \varepsilon^{2} z\left(\left.\frac{\partial v}{\partial n}\right|_{+}-\left.\frac{\partial v}{\partial n}\right|_{-}+\left.\frac{\partial z}{\partial n}\right|_{+}-\left.\frac{\partial z}{\partial n}\right|_{-}\right)\right\} \\
& -\int_{0} f^{2}\left(v z^{3}+(3 / 2) z^{4}\right)
\end{aligned}
$$

implies that the Fréchet derivative vanishes on this subset of $H$ when

$$
\left.\frac{\partial v}{\partial n}\right|_{+}=\left.\frac{\partial v}{\partial n}\right|_{-}
$$

and the general theory of elliptic equations implies that since $v$ and $\nabla v$ are continuous across $L$ equation $(1,1 \mathrm{a})$ is satisfied on $L$. The argument reverses easily. Thus it follows:

LEMMA 1. For any norm, defined on a family of lines, which guarantees completeness in the properties necessary for the domain so defined to possess a positive solution, successful testing with a small ball of lines suffices to guarantee the existence of a solution to both the variational problem and the partial differential equation.

It follows immediately that:

LemMa 2. A line $L$ is a nodal line of a solution of equation $(1,1 \mathrm{a} \& \mathrm{~b})$ if and only if

$$
\mathscr{E}^{\prime}(L)=0
$$


There is no advantage to be gained from using this form over Lemma 1 unless the considerations can be removed from $H$. How this might be achieved will now be discussed.

\section{Approximate variational principle}

In view of the total lack of knowledge of where the nodal lines can be located, heuristic arguments are useful as a guide. A "natural" physical argument, for which there is no theoretical basis, is that the processes of taking the Fréchet derivative and the limit of small $\varepsilon$ commute. Thus we were led to use the known asymptotic properties of the positive solutions to estimate the energy functional in an attempt to surmise the location of nodal lines. The first step in such an approach is to obtain an expression for the value of the functional in terms of the asymptotic values of the positive solution for a domain. A convenient form of a uniform approximation to $P(\mathbf{x}, D)$ can be obtained as (see Appendix)

$$
P(\mathbf{x}, D)=g \tanh (\zeta / \sqrt{2})
$$

where

$$
\zeta=q / \varepsilon
$$

and $q$ vanishes on the boundary and satisfies

$$
(\nabla q)^{2}=f^{2} g^{2}
$$

This may be verified to satisfy the partial differential equation $(1,1 \mathrm{a} \& \mathrm{~b})$. Substitution of equations $(3,1)$ into the expression for $E$ in equation $(1,2)$ yields

$$
E(P)=(4 / 3) \sqrt{2} \varepsilon \int_{L} f g^{3} d s+a \text { constant }+O(\varepsilon) .
$$

Thus the leading form of the asymptotic behaviour is obtained and, if a variational argument is applied to this as a matter of hope, the problem reduces to a classical problem, namely seeking lines $L$ which render stationary the function $\mathscr{V}$ defined by

$$
\begin{aligned}
\mathscr{V}(L) & =\int_{L} f g^{3} d s \\
& =\int_{L} h d s \quad \text { (defn.), }
\end{aligned}
$$

where the latter portion serves to define $h$. For a closed line $L$ in the interior of $D$, there are no difficulties with regard to end-points. But where $L$ intersects the boundary the domain will have corners or worse and the use of equations $(3,1)$ 
near there is certainly not justified. However because the positive solution is the global minimiser, any approximate form substituted in the functional over-estimates the functional. Replacement of the form $(3,1)$ in the corners by some form of near-conical patch will contribute a term which is close to $O\left(\varepsilon^{2}\right)$ and hence certainly negligible in comparison with the order to which $E$ has been evaluated. Thus equation $(3,3)$ still is appropriate.

The 'approximate' form of variational principle is a classical weighted geodesics problem about which a great deal is known. The Euler-Lagrange equation determines a second-order ordinary differential equation, which is in essence a determination of the curvature of the geodesic. For a line joining fixed end-points, the geodesic is the sole critical point and a minimum of the functional $\mathscr{V}$. This holds whether the line is a loop or otherwise. The boundary conditions for the Euler-Lagrange equation come from varying the end-points. For a closed loop the location of a solution utilises the closing of the geodesic and making the functional stationary is comparison with neighbouring loops.

For the case of end-points lying on the boundary, each end-point is located so that the functional is stationary with respect to variation of the end-point. An implication of this is that the line $L$ must be orthogonal to the boundary at the intersection. Now this must also be true for a nodal line of any solution where the boundary is smooth. For if it were not, there would be no satisfactory solutions in contiguous corners. The behaviour of solutions to elliptic equations with 'natural' corners (as distinguished from pathological) can be approximated in a series of terms which, if the boundaries were locally straight, would be algebraic powers of distance from the corner with coefficients functions of angle in the corner. For curved boundaries, these terms need iterative correction, but this does not modify the nature of the solution behaviour. The specific powers are determined as eigen-values, related solely to the corner angle, and if these angles are not equal it is not possible for the normal derivative to be continuous across $L$ in the neighbourhood of the corner. Nor is it likely that $L$ is orthogonal to the boundary but incident on the boundary at some distance from the natural continuation of the geodesic relevant in the interior. For if the nodal line diverted near the boundary and thus generated a narrow tongue, the contribution of such a tongue is almost certain to render the Fréchet derivative non-zero. The plausible conjecture is that despite the inadequacy of the corner estimates, nodal lines are likely (?) to lie near to stationary weighted geodesics.

The guesses above indicate a close connection between nodal lines and stationary geodesics. Some care is necessary even if the indications are generally correct. The existence of a stationary value in an approximation may not imply the existence of one in the exact form. A curve with a stationary point of inflexion can be perturbed into a curve without a stationary point. So can a maximum or 
minimum with a curvature small in comparison with the perturbation applied. The obvious conclusion is that any attempt at a proof of a connection between nodal lines and stationary geodesics must invoke assumptions that the stationary behaviour of the geodesics is sufficiently "decisive".

The question about saddle-points has also to be faced. Because the stationary value for 'interior variations' is always a minimum, only those geodesic problems which are also minimal for boundary variations can be associated with minima of the original variational problem set in $H$. Geodesic problems with local minima are rather rare. For example with a unit weight applying, the minor axis of an ellipse is not a minimum of the functional - translation of the minor axis contradicts this. It takes a geometric shape like a dumb-bell to lead to a minimum. Thus if the geodesic is to provide a guide to the results then in most circumstances the functional $E$ will possess saddle-points, and some form of cartesian product decomposition is needed to separate boundary and interior variations. Such a decomposition in $H$ is awkward and certainly the form using a selected set of lines has many attractions.

\section{Asymptotic limits of nodal lines}

THEOREM 1. (A) For each $\varepsilon$, with $0<\varepsilon<\varepsilon_{0}$, let a solution of equations $(1,1 \mathrm{a} \& \mathrm{~b})$ exist with a simple nodal line $L_{\mathrm{e}}$ of either character (Figure 1$)$. Let the curvature of these lines be bounded independently of $\varepsilon$. Let a line $L_{0}$ exist such that, if $\mathbf{x}$ is on $L_{0}$ and $\mathbf{y}$ is on $L_{\varepsilon}$ and both lie on a common normal to $L_{0}$,

$$
\operatorname{limit}_{\boldsymbol{\varepsilon} \rightarrow 0} \sup |\mathbf{x}-\mathbf{y}|=\mathbf{0} \text {. }
$$

Then $L_{0}$ is a weighted geodesic, but not necessarily a stationary one in the terminology of Pars [5].

(B) Let the end-points of $L_{\varepsilon}$ be $\left(\mathbf{p}_{e}, \mathbf{q}_{\varepsilon}\right)$ and let $L(\mathbf{p}, \mathbf{q})$ denote any line through neighbouring end-points $(\mathbf{p}, \mathbf{q})$. Define $M(L(p, q))$ by

$$
M[L(\mathbf{p}, \mathbf{q})]=\inf _{L(p, q)} \varepsilon^{-1 \mathscr{E}}[L(\mathbf{p}, \mathbf{q})]
$$

For small $r$, where

$$
\mathbf{r}=\left(\mathbf{p}-\mathbf{p}_{\boldsymbol{e}}, \mathbf{q}-\mathbf{q}_{\boldsymbol{e}}\right),
$$

define a quadratic estimator $Q$, by

$$
Q(\mathbf{r})=M[L(\mathbf{p}, \mathbf{q})]-M\left[L\left(\mathbf{p}_{\varepsilon}, \mathbf{q}_{\varepsilon}\right)\right]+O\left(r^{3}\right) .
$$

Assume that the eigenvalues associated with the quadratic form $Q$ are bounded away from zero uniformly.

Then $L_{0}$ is a stationary weighted geodesic of $\mathscr{E}$ and the nature of the stationary value is inherited from $Q$. 
Proof. Since $L_{0}$ is a nodal line it follows from Lemma 1 that equation $(2,8)$ holds. But for interior points away from the corner these normal derivatives can be estimated from the asymptotic solution. The assumption is made that, to the algebraic order wanted, the effects of the corner (if one is involved) tend to zero with $\varepsilon$. A more detailed analysis of the asymptotics is required to obtain the accuracy needed. These are developed in the Appendix, where it is shown that the normal derivatives will be continuous across $L$ if

$$
\kappa=h^{-1} \frac{\partial}{\partial n}(h) \text {. }
$$

Here $\kappa$ is the curvature of the nodal line, and an error of $O(\varepsilon)$ has not been displayed. This equation is the Euler-Lagrange equation for the functional $\mathscr{V}$. In the limit of $\varepsilon$ tending to zero the error term suppressed in equation $(4,1)$ vanishes and equation applies to $L_{0}$. Thus the limiting nodal line satisfies the EulerLagrange equations and so is a weighted geodesic.

Note. There is no suggestion in this proof under the conditions of part (A) that $L_{0}$ need be a stationary geodesic.

It seems essential to invoke the conditions of part (B) in order to obtain the stronger result. The factor $\varepsilon^{-1}$ introduced in front is a matter of convenience in presentation so that the terms do not all tend to zero in the limit. Now,

$$
\begin{aligned}
M[L(\mathbf{p}, \mathbf{q})] & -M\left[L\left(\mathbf{p}_{0}, \mathbf{q}_{0}\right)\right] \\
= & M[L(\mathbf{p}, \mathbf{q})]-M\left[L\left(\mathbf{p}_{\varepsilon}, \mathbf{q}_{\varepsilon}\right)\right]-M\left[L\left(\mathbf{p}_{\varepsilon}, \mathbf{q}_{\varepsilon}\right)\right]-M\left[L\left(\mathbf{p}_{0}, \mathbf{q}_{0}\right)\right] \\
= & Q(r)+O\left(r^{3}\right)+\int v \kappa d s .
\end{aligned}
$$

As $\varepsilon$ tends to zero the difference between the functional evaluated on neighbouring lines and the limiting line is dominated by the quadratic term so that the result of part (B) follows.

Thus it is necessary for a nodal line to lie close to the stationary geodesic for non-pathological (continuity in the limit of small $\varepsilon$ ) behaviour when the stationary value of the geodesic is quadratically decisive. For purposes of proof the assumption is placed on the functional. But since the decisiveness is inherited there is a trivial corollary.

COROLlary. If $L_{0}$ is a weighted geodesic which is not decisive then it cannot be the limit of a family of nodal lines which are decisive.

As a consequence any nodal line, for which the nature of the critical point in the space $H$ is determined quadratically, is close to a decisive stationary weighted geodesic asymptotically. 
Comment. For an equation in which the function $h$ is constant, the geodesics are straight lines and any extremum of the weighted geodesic length is associated with the intercept on the boundaries. Thus in a rectangle all lines parallel to the sides are of equal geodesic length and the methods discussed here are of little predictive value. The present ideas are most powerful for boundaries without symmetry and for functions $h$ with spatial heterogeneity.

The result, which one would really like to establish, is that there is a nodal line close to such decisive stationary weighted geodesics. The general proof has eluded us even though we believe the result to be true. The essential difficulty arises from the fact that it is necessary to establish a location for the nodal line displaced by some distance from the marked geodesic. But the proofs have their basis in the variational problem set in $H$. In order to make the connection, estimates of $v$ in terms of the displacement are needed. But the origin of estimates for $v$ lies in equations $(2,3)$ and as has already been observed no useful such estimates are available. Yet it is a reasonable surmise that this equation does not lie close to the spectrum and so normal properties will hold even if they are difficult to prove. The surmise is based on the argument that if the spectrum was a real, as distinct from a technical, problem, the robust properties of the positive solutions would not hold.

Thus we are led to suggest the following result.

Conjecture. For small enough $\varepsilon$, corresponding to every decisive stationary weighted geodesic $L_{0}$, there exists a solution of the type displayed in equation $(2,4)$ with the nodal line close to $L_{0}$.

\section{Analogous differential equation}

For the corresponding ordinary differential equation

$$
u^{\prime \prime}+f^{2}\left(g^{2}-u^{2}\right) u=0, \quad 0<x<1,
$$

and homogeneous boundary conditions for $u$, a similar approach can be used with almost identical indications. The corresponding functional, for which equation $(5,1)$ is the Euler-Lagrange equation, is

$$
E(w)=\int_{0}^{1}\left[\left(w^{\prime}\right)^{2}+f^{2}\left(g^{2}-w^{2}\right)^{2} / 2\right] .
$$

The approximating functional is similarly

$$
\mathscr{V}(l)=\left.f g^{3}\right|_{l}=\left.h\right|_{l} \quad \text { (by defn.) }
$$


where $l$, representing a point, replaces the line $L$ in the plane case. Rather than use the corresponding term nodal point (or node) we choose the word zero for preference. Thus the suggested locations of the zeros of the non-positive solutions lie close to the extrema of $h$. It is possible to prove many of the results, previously only conjectured, and to establish further results.

The greater body of techniques for ordinary differential equations enables other approaches to be used even though the problem of extreme sensitivity remains. Thus the positive solution can be shown to have the following property.

LEMMA 3. Let $q(y)$ denote the unique positive solution on $(0, y)$, and let $d$ be the value of $q^{\prime}(0)$ for that solution. Then $d$ is a monotone increasing function of $y$.

Proof. Close to the small value of $y$, at which a positive solution bifurcates from $y=0, d$ is an increasing function of $y$. If, for larger $y$, it decreased somewhere, there would be two values of $y$ corresponding to the same value of $d$. But the initial values $u(0)=0, u^{\prime}(0)=d$ determine a unique solution so that the larger value of $y$ is not a first zero and the corresponding solution is not positive.

Comment. The range of values of $d(y)$, if $y$ is bounded away from 0 , independently of $\varepsilon$, is of very small order in $\varepsilon$. Any solution of equation $(5,1)$ which follows the curve $u=g$ for any length and starts from $x=0$ for example, is described asymptotically in $\varepsilon$, by the same approximation to any algebraic power in $\varepsilon$. Thus the range of values of $d$ relevant for consideration is transcendentally small in $\varepsilon$. This is the source of the difficulty in attempting to establish asymptotic results as estimates, good enough to resolve the solution behaviour.

It is convenient to take advantage of the Liouville transformation to reduce the complexity of the calculations. Thus introduce a new independent variable $\zeta$, defined by

$$
\zeta=\varepsilon^{-1} \int_{0}^{x} f g,
$$

and note that $x$ and $\zeta$ are in 1-1 correspondence, so that a function of either variable is closely associated with a function of the other. It is often convenient not to bother to draw a distinction in notation. The dependent variable is scaled to $g$ by introducing $U$ defined by

$$
u=g U .
$$

In terms of these new variables equation $(5,1)$ becomes

$$
U^{\prime \prime}+U\left(1-U^{2}\right)+\varepsilon U^{\prime} H_{x} / m+\varepsilon^{2} U J / m^{2}=0
$$

where

$$
H=\ln h, \quad J=g_{x x} / g, \quad m=f g .
$$


Here subscript $x$ denotes ordinary differentiation with respect to $x$ in contrast to the use of ' to denote differentiation of the corresponding function with respect to $\zeta$.

An energy integral can be obtained by multiplying equation $(5,6)$ by $U^{\prime}$ and integrating with respect to $\zeta$. The result is

$$
\begin{aligned}
0= & {\left[U^{\prime 2} / 2+U^{2} / 2-U^{4} / 4\right]_{0}^{\zeta}+\varepsilon \int_{0}^{\zeta} U^{\prime 2} H_{x} / m d \zeta } \\
& +\left[U^{2} J \varepsilon^{2} / m^{2}\right]_{0}^{\zeta}-\varepsilon^{2} \int_{0}^{\zeta} U^{2} D_{\zeta}\left(J / m^{2}\right) d \zeta .
\end{aligned}
$$

The derivation of this equation requires the assumption that $g$ is thrice differentiable, and $f$ once, in a sense making the operations valid. This will henceforth be assumed.

THEOREM 2. Let (1) a solution exist with a single interior zero $x_{0}$,

(2) a constant $K$ exist such that

$$
\operatorname{Max}\left[H_{x}, J / m,\left(J / m^{2}\right)\right]=K,
$$

(3) a positive constant $b$ exists such that

$$
\left|H_{x}\right|>b \text {. }
$$

Then, for sufficiently small $\varepsilon, x_{0}$ lies within an $\varepsilon$-neighbourhood of that end of the interval at which $H$ takes its maximum value. Further $x_{0}$ tends to the endpoint as $\varepsilon$ tends to zero.

Proof. Under the assumptions made the energy integral can be arranged in the form

$$
U^{\prime 2}-\left(1-U^{2}\right)^{2} / 2=-1 / 2+\left[U^{\prime}\left(x_{0}\right)\right]^{2}-2 \int_{x_{0}}^{x} U^{\prime 2} H_{x} d x+O(\varepsilon) .
$$

If the point $x_{0}$ is not close to an endpoint, since the solution on each interval behaves like the appropriate positive solution, the integral on the right-hand side can be estimated in terms of $\int_{\zeta_{0}}^{\zeta} U^{\prime 2} d \zeta b$.

Further the solutions may be bounded on either side by solutions of the approximate energy form

$$
Y^{\prime 2}-\left(1-Y^{2}\right)^{2} / 2=c
$$

for suitable constants $c$. But the behaviour of such solutions is known to be:

if $c>0$, then $|Y|$ increases indefinitely;

if $c<0$, then $Y^{\prime}$ changes sign and the solutions are periodic with a period becoming large for $c$ tending to zero.

Thus it may be shown that, on the side of $x_{0}$ on which $H$ is larger, $U^{\prime}$ changes sign and $U$ returns to zero at a value of $\zeta$ differing by $O(1)$ from that at $x_{0}$ (but becoming large as $b$ tends to zero). The result of the theorem follows. 
COROLlaRY. If the function $h$ is not constant then any solution which has just one zero in the interior, with that zero not in an e-neighborhood of the boundary, can have that zero only close to a local maximum or minimum of $h$.

A number of interesting inferences follow from results used in the proof. The integral term

$$
\int_{x_{0}}^{x} U^{\prime 2} H_{x} d x
$$

is necessarily positive in the neighbourhood of a local minimum of $h$. If the solution has another zero at a neighbouring point $x_{1}$ the corresponding energy constant will be less for $x_{1}$ than it was for $x_{0}$. The solution will therefore have to possess a further zero. This argument can be repeated while $H_{x}$ retains its sign. Even if a local maximum occurs, reattachment to the curves $\pm g$ will be delayed. It would appear that the use of singular perturbation techniques appropriate to non-linear oscillation problems with slowly varying coefficients may be used to locate approximately the point of reattachment.

There is no such condition applicable to a local maximum of $h$. It follows therefore that solutions with a moderate number of zeros must have them clustered about a local maximum or at the boundary where $h$ is largest.

The question as to whether there are solutions with interior zeros is as yet unanswered. An example of existence results which can be proved using this approach is the following theorem.

THEOREM 3. Let $x_{0}$ be a decisive global minimum of $H$ in the interior of $(0,1)$. Let no other extrema exist.

Then, for $\varepsilon$ sufficiently small, there exists a solution with just one zero and that zero tends to $x_{0}$ as $\varepsilon$ tends to zero.

Proof. Choose an arbitrary point, $\xi$, in $(0,1)$ and define a function $W$ by

$$
\begin{aligned}
W & =P_{1}(x, \xi), \quad 0<x<\xi, \\
& =-P_{2}(x, \xi), \quad x>\xi,
\end{aligned}
$$

where $P_{1}$ and $P_{2}$ are the positive solutions on the respective domains. As previously, this will be a solution of the desired form if, and only if,

$$
P_{1}^{\prime}(\xi, \xi)=-P_{2}^{\prime}(\xi, \xi)
$$

For each $\xi$ there is a uniquely determined $P^{\prime}(0)$ and $P^{\prime}(1)$ generating these solutions, and their values are limited, at an algebraic order, to a value calculable using asymptotic methods. For the positive solutions, it follows from the energy 
integral that

$$
\left[W^{\prime}(\xi)\right]^{2}-\left[W^{\prime}(0)\right]^{2}=-\varepsilon \int_{0}^{\zeta_{0}} W^{\prime 2} H_{x} / m d \zeta-\varepsilon \int_{0}^{\xi} W^{2} D_{x}\left(J / m^{2}\right) d x,
$$

where $\zeta_{0}$ corresponds to the point $\xi$.

A similar result is obtained on $(\xi, 1)$. Then $D(\xi)$, defined by,

$$
D(\xi)=\left[W^{\prime}\left(\xi_{+}\right)\right]^{2}-\left[W^{\prime}\left(\xi_{+}\right)\right]^{2},
$$

is given by

$$
D(\xi)=\left[W^{\prime}(0)\right]^{2}-\left[W^{\prime}(1)\right]^{2}+2 \int_{0}^{1} W^{\prime 2} H_{x} d x-2 \varepsilon^{2} \int_{0}^{1} W^{2}\left(J / m^{2}\right)_{x} d x .
$$

The distinguishing notation $W(x, \xi)$ and $W\left(x, x_{0}\right)$ is introduced and $[D(\xi)-$ $D\left(x_{0}\right)$ ] calculated. Note that

(1) A similar calculation for $\Delta \partial v / \partial n$, made in Section 4 , can be translated to the present case and yields

$$
D\left(x_{0}\right)=0\left(\varepsilon^{2}\right) .
$$

(2) The comment following Lemma 3 implies that both,

$$
W^{\prime}\left(0, x_{0}\right)-W^{\prime}(0, \xi)=O\left(\varepsilon^{2}\right)
$$

and

$$
W^{\prime}\left(1, x_{0}\right)-W^{\prime}(1, \xi)=O\left(\varepsilon^{2}\right),
$$

hold, provided that $(\xi \gg \pi \varepsilon / m(0))$ and $((1-\xi) \gg \pi \varepsilon / m(1))$. (No positive solution exists on too small an interval.)

Thus the expression for $\left[D(\xi)-D\left(x_{0}\right)\right]$ yields the approximation

$$
D(\xi)=2 \int_{0}^{1} H_{x}\left\{\left[W^{\prime}(0, \xi)\right]^{2}-\left[W^{\prime}\left(0, x_{0}\right)\right]^{2}\right\} d x+O\left(\varepsilon^{2}\right) .
$$

In the neighbourhoods of $0, x$, and 1 the approximation,

$$
\left[W^{\prime}(x, *)\right]=(1 / \sqrt{2}) \operatorname{sech}[\zeta-\zeta(*)]+O(\varepsilon),
$$

holds if the appropriate replacement is made for *. If $\xi$ is held sufficiently separated from $x_{0}$, the integral is easily estimated to show that

$$
D(\xi)=\varepsilon C H_{x}(\xi) / m(\xi)+O\left(\varepsilon^{2}\right), \text { for } \varepsilon^{-1}|x-\xi| \gg 1 .
$$

Here

$$
C=\frac{1}{2} \int_{-\infty}^{\infty} \operatorname{sech}^{4} \zeta d \zeta
$$

Since $D(\xi)$ is continuous and takes opposite signs on either end of a small interval about $x_{0}, D$ vanishes at some value $\xi_{1}$ of $\xi$ and $W\left(x, \xi_{1}\right)$ is a solution with one zero. 
The proof is completed noting that if $\xi_{0}$ does not tend to $x_{0}$ with $\varepsilon, D\left(\xi_{1}\right)=0$ is inconsistent with equation $(5,9)$.

Comments. (1) The proof does not rule out the possibility that there is more than one location for the sole zero.

(2) This line of proof can be extended systematically to cover many other cases. To show the existence of a solution with just one zero near another allowed location, a similar argument can be mounted. Solutions with one zero can then be estimated to the accuracy needed, namely that needed for the positive solutions. Solutions with two zeros are then treated as the adjunction of a positive solution on one interval with a solution with one zero on the other interval. Similar arguments using a variable location $\xi$ for the joining point are then applied. The only real change comes in dealing with those cases where the distance between zeros tends to zero with $\varepsilon$. The use of the autonomous form of the energy integral allows suitable bounds to be obtained.

(3) No attempt has been made to justify the formal estimates for the positive solutions. This hardly seems necessary, for a write down to this much simpler problem of Berger and Fraenkel's derivation of the results for the partial differential equation would provide a validation.

\section{Mathematical implications}

At least for the ordinary differential equation, the results which were merely conjectured for the partial differential equation are found to be true. It is convenient to use the language analogous to the partial differential equation problem in the context of the ordinary differential equation. There do exist solutions with isolated nodal lines close to stationary weighted geodesics and, if the function $h$ does not contain any flat parts, then they are the only possible locations for nodal lines of solutions. All other solutions with several nodal lines have them clustered around the maximal weighted geodesic or the boundary. There is good reason to add a similar addendum to the conjecture about the partial differential equation. Suppose such were to have a solution with two distinct nodal lines, partitioning the domain into three or four sub-domains. Then on each sub-domain, the appropriate local positive solution applies and the problem transforms, as before, to a variational problem posed on lines. The indications obtained from $h$ seem to have been so appropriate that there does seem to be value in examining the approximate asymptotic functional and this implies that each line separately must independently be a stationary weighted geodesic. There are a strictly limited number of such and therefore the clustering effect is indicated for the plane case as well. 
Wherever results are established, the function $h$ plays a role of considerable significance and it is a striking combination of $f$ and $g$ which is difficult to associate with the equation other than from the approximate functional. While $h$ does arise through the Liouville variable, which finds use in approximate asymptotics of partial differential equations, no exact theory appears to have been based on it other than for ordinary differential equations. This suggests that there is a deeper reason for the significance of $h$.

Moreover, as arises in the proof of Theorem I, the Fréchet derivative, $E^{\prime}$, vanishes asymptotically merely if a potential nodal line has geodesic curvature. The use of the alternative form using the approximate functional, $\mathscr{V}(L)$, proves much more powerful. One is tempted to surmise that, where it provides discrimination between lines, the functional $\mathscr{V}$ controls the properties of $E$. Perhaps it may be a more fruitful approach to seek to formulate this abstractly than to become engrossed in the very detailed calculations of each particular example.

In conclusion it may be mentioned that the general motivation transfers with very little modification to more than two independent variables. The only change is to replace geodesic by minimal surface until one comes to the determination of the latter objects. Otherwise similar results appear to apply there.

\section{Biochemical diffusive system applications?}

The ideas in the earlier sections may be helpful in attempts to define the role of reactive-diffusive mechanisms in the control of pattern formation in biological systems. There are many species with very distinctive and complex surface patterns with a specification requirement that would be 'expensive' to code genetically. An alternative mechanism has been suggested. It is that the patterns arise with the boundaries between colours being defined as regions of significant diffusion between different equilibrium states for a chemical system. Murray, [3], [4] has used a simple numerical model to show that the patterns of different species of zebra could be accounted for by this mechanism. The results are strongly indicative but have been subject to criticism on the grounds of the use of special chemical kinetics of a low order system. The case would strengthened if it could be shown that such pattern determination was a more general outcome in systems of reactions of very complex nature and thus necessarily of high mathematical order. The problem is to make testable predictions without there being any strong hope of possessing reliable knowledge of the chemistry let alone the kinetics.

The mathematical class of reactive diffusive systems we consider are not ones for which it would be possible to provide empirical evidence that such kinetics exist. But they have the significant property that the patterns that develop are 
fixed by the geometry of the surface on which they develop. Species pattern differentiation could thus be explained in terms of the timing of the initiation and termination of a reaction in relation to shape and size of the surface involved. Significantly the pattern is predictable, using the present results, from the observed number of chemical equilibrium states and observations of the surface geometry at the stage of pattern formation. The results therefore provide a framework against which specific predictions could be tested. We see this as the essential justification for the development of the model.

The partial differential equation $(1,1$, a) can be considered as an archetype equation for a system for which there are two stable outcomes, the positive and negative solutions, and for which a variational principle is available. If the equation, not the solution, were spatially homogeneous than no particular boundaries between regions of different outcome would be determined. It is the dependence of the coefficients on $\mathbf{x}$ which serves to limit the patterns in such a remarkable fashion. However the model equation used earlier, and particularly in the form of Berger and Fraenkel [1], is inappropriate in that the outcome is spatially dependent. It is however reasonable to include spatially dependent diffusion terms on a curved surface layer of possibly varying thickness. In such circumstances superficial diffusion will be described by a spatially varying elliptic operator replacing the Laplacian used above. The chemical state can be described by specifying the amount of each chemical involved including all intermediate products. The vector $\mathbf{u}$ will be used to denote the chemical state. In the absence of diffusion the chemical kinetics can be described by

$$
D_{t} \mathbf{u}=-\mathbf{R}(\mathbf{u})
$$

where $\mathbf{R}$ denotes the reaction rate vector and it is assumed that temperature changes are not significant. The order of both vectors is denoted by $N$, the value of which is of little concern. It is further assumed that there are a number of equilibrium states $\mathbf{U}_{\alpha}$ at which $\mathbf{R}$ vanishes and all eigenvalues of the Fréchet derivative of $\mathbf{R}$ with respect to $\mathbf{u}$ are positive, thus ensuring local stability of the equilibrium state.

A generated model for the combined effects of reaction and diffusion is thus given by

$$
D_{t} u_{l}=k_{l} q D_{l}\left[A_{l j}(\mathbf{x}) D_{l} u_{l}\right]-R_{l} .
$$

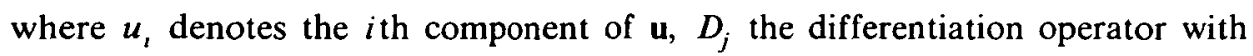
respect to the $j$ th surface coordinate and $q$ and $A_{i j}$ the standard coefficients of the representations of the Laplacian in surface coordinates, modified for thickness if considered necessary. The diffusion coefficient for each component is given by $k_{l}$. All diffusion coefficients are assumed to be small in comparison on the scale 
defined by the surface pattern length scale and a time scale defined by all reaction ates when the system is not in equilibrium. Such an assumption would not be justified where the transition from one equilibrium state to another was gradual.

In order to generalise the earlier results, a variational formulation of this system is needed to permit the previous ideas to be used. We make the simple assumption that $\mathbf{R}$ is the gradient of a potential $V$ with respect to $\mathbf{u}$, so that equation $(7,2)$ is the Euler-Lagrange equation for critical points of the functional

$$
E(\mathbf{u})=\sum A_{i j}(\mathbf{x}) D_{l} D_{j} u_{l} k_{l}+q V .
$$

It may be observed that the function class on which the criticality is to be tested depends on the boundary condition for the whole domain. Any of the standard boundary conditions for diffusive systems may apply and the method will work. The most likely one is no transport of a component through any external boundaries.

If a line $L$ is the boundary between regions where different equilibrium states prevail and $\mathbf{n}$ is the insurface normal to $L$ and $z$ is a coordinate along the normal measured from $L$ then equation $(7,2)$ is approximated by

$$
Q(\mathbf{x}) k_{l} D_{n} D_{n} u_{l}=-q D u_{l} V
$$

where

$$
Q(\mathbf{x})=A_{\imath \jmath}(\mathbf{x}) n_{\imath} n_{\jmath}
$$

This system is a Hamiltonian and there is a range of possible methods for solution. A solution means the determination of a path in u-space $u=\mathbf{U}(z)$, joining the two equilibrium states, such that equation $(7,4)$ is satisfied. If the end points are denoted by $\mathbf{U}_{\alpha}$ and $\mathbf{U}_{\beta}$ to maintain the notation of the earlier context, then the "energy" integral of equation $(7,5)$ is

$$
1 / 2 Q(\mathbf{x}) \sum k_{l}\left[D_{z} U_{l}(z)\right]^{2}=V(\mathbf{U})-V[\mathbf{U}(z)] .
$$

In order that the path should lead to the other equilibrium state it is necessary that

$$
V\left(\mathbf{U}_{\alpha}\right)=V\left(\mathbf{U}_{\beta}\right)
$$

In terms of a parameter $U$ fixing position on the path in $u$-space, equation $(7,7)$ may be written as

$$
D_{z} U(z)=[Q(\mathbf{x}) / q] P_{l}(U)
$$

for some function $P$. The variational integral can now be evaluated in principle and the mathematical forms are such that

$$
E(L)=\text { const. } \oint[q Q(\mathbf{x})]^{1 / 2} d s .
$$


All the details of the chemistry have been absorbed into the multiplicative constant, as are the details of the equilibrium states. All that is required is that transition between the various end states is possible across thin regions of significant diffusion. Then any portion of a boundary between different states has to be a weighted geodesic line for the variational problem defined by the functional in equation $(7,7)$. For given surfaces and boundary conditions, these are limited and considerable predictions about the patterns are obtainable solely in terms of the surface geometry, and possible the boundary conditions, at the time when the pattern was determined. This isolation of the factors controlling patterns in the class of reactions considered is very striking. It is completely dependent on the spatial variability of the coefficients. Whether it is restricted to systems which have the gradient property for their rate vector is a matter of conjecture. But it is apparent that if it were then there could be a selective mechanism favouring such types of chemistry in species development. What is of more immediate consequence is the possibility of more intensive testing of the underlying hypothesis concerning the reactive-diffusive generation of patterns.

The discussion above is based on equilibrium considerations and the possible patterns could arise only from initial conditions sufficiently varying in space to make the patterns possible. We comment that reactive-diffusive systems also generate travelling wave solutions and this is a possible source of suitable initial states for the reactions leading to the equilibrium states we have been discussing.

\section{Appendix}

The discontinuity in the normal derivative across a nodal line can be calculated, to the accuracy required here, from an approximation for the derivatives of the positive solution accurate to $O(1)$. Such an approximation is obtained using formal approximations. The method of proof of Berger and Fraenkel [1] may be used to justify any expression reducing the error in the partial differential equation sufficiently, so that the results obtained by the present method may be justified. The following modification of the Liouville transformation achieves a satisfactory expression simply.

Let $v[\mathbf{x}]$ be a positive function, defined in an order one neighbourhood of the boundary, by the first order differential equation

$$
[\nabla v]^{2}=f^{2} g^{2}
$$

the boundary condition

$$
v(x)=0, \quad \mathbf{x} \in \partial D .
$$


Outside this neighbourhood, $v$ is patched smoothly over the remainder of the domain. A boundary layer variable $\zeta$ is defined by

$$
\zeta=v(\mathbf{x}) / \varepsilon,
$$

a solution is sought in the form

$$
u=g(\mathbf{x}) U(\mathbf{x}, \zeta, \varepsilon) .
$$

As a consequence of the equation (A1), equation $(1,1)$ becomes

$$
\begin{aligned}
0= & U_{5 \zeta}+U\left(1-U^{2}\right)+\varepsilon^{2} f^{-2} g^{-3} \nabla^{2}(U g) \\
& +\varepsilon f^{-2} g^{-3}\left\{2\left[\nabla\left(U_{\zeta} g\right) \cdot \nabla v\right]+U_{\zeta} g \nabla^{2} v\right\} .
\end{aligned}
$$

Where now the operator is calculated with the variable $\zeta$ being treated as independent of $\mathbf{x}$. The results of substituting the formal series,

$$
U=U_{0}+\varepsilon U_{1}+O\left(\varepsilon^{2}\right),
$$

into the differential equation and equating powers of $\varepsilon$ to zero are

$$
\begin{gathered}
U_{0 \zeta \zeta}+U_{0}\left(1-U_{0}^{2}\right)=0 \\
0=U_{1 \zeta \zeta}+\left(1-3 U_{0}^{2}\right) U_{1}+f^{2} g^{-3}\left[2(\nabla g) \cdot(\nabla v)+g \nabla^{2} v\right] U_{0 \zeta} .
\end{gathered}
$$

The appropriate boundary conditions are

$$
U_{i}(0)=0, \quad U_{i}^{\prime}(\infty)=0, \quad i=1,2 .
$$

The solution of equation (A5) is easily shown to be

$$
U_{0}(\zeta)=\tanh (\zeta / \sqrt{2})
$$

As the derivative, $U_{0}^{\prime}$, is a solution of the homogeneous equation satisfied by $U_{1}$, the boundary value problem posed by equations (A6\& 7) will have a solution if, and only if,

$$
0=\int_{0}^{\infty}\left(U_{0 \zeta} U_{1 \zeta}-U_{1} U_{0 \zeta \zeta}\right)_{\zeta} d \zeta+f^{-2} g^{-3}\left\{2(\nabla g) \cdot(\nabla v)+g \nabla^{2} v\right\} \int_{0}^{\infty} U_{0 \zeta}^{2} d \zeta
$$

which is equivalent to

$$
U_{1 \zeta}(\mathbf{x}, 0)=f^{-2} g^{-3}\left[2(\nabla g) \cdot(\nabla v)+g \nabla^{2} v\right] \int_{0}^{\infty} U_{0 \zeta}^{2} / U_{0 \zeta}(0) .
$$

Thus, on the boundary, the value of the normal derivative is given by

$$
\mathbf{n} \cdot \nabla u=\left.\varepsilon^{-1} \mathbf{n} \cdot \nabla v\left\{U_{0 \zeta}+\varepsilon U_{1 \zeta}\right\}\right|_{\zeta=0}+\mathbf{n} \cdot \nabla g U_{0}(0)+O(\varepsilon)
$$

When this result is applied on either side of a nodal line, a number of terms cancel when allowance is made for the opposite signs of the normal. The only terms contributing to the discontinuity of the normal derivatives are those involving the $[\cdots]$ term in equation (A8) and these may be arranged as

$$
\text { const. } h^{-1} \nabla \cdot(h \mathbf{n}) \text {. }
$$


Thus the condition, accurate to unit order, for there to be no discontinuity of the normal derivative is

$$
k+h^{-1} \frac{\partial h}{\partial n}=0
$$

where $k$ is the curvature of the boundary.

\section{References}

[1] M. S. Berger and L. E. Fraenkel, "On the asymptotic solution of a non-linear Dirichlet problem," J. Math. Mech. 19 (1969-70), 553-585.

[2] P. J. Budden and J. Norbury, "A nonlinear eigenvalue problem," J. Inst. Math. Appl. 24 (1979), 9-33.

[3] J. D. Murray, "Prepattern formation mechanism for anımal coat markings," J. Theoret. Bıol. 88 (1981), 161-199.

[4] J. D. Murray, "On pattern formation mechanism for lepidopheran wing patterns and mammalian coat markings," Philos. Trans. Roy. Soc. London Ser. B 295 (1981), 473-496.

[5] L. A. Pars, An tntroduction to the calculus of variations (John Wiley and Sons Inc, New York, 1962). 